\title{
On Locating-Dominating Codes in Binary Hamming Spaces
}

\author{
Iiro Honkalal] and Tero Laihonen! and Sanna Ranto
}

Department of Mathematics and Turku Centre for Computer Science, University of Turku, FIN-20014 Turku, Finland.

\{honkala, terolai, samano\}@utu.fi

received Nov. 5, 2002, revised Dec. 3, 2003, accepted Jan. 6, 2004.

Locating faulty processors in a multiprocessor system gives the motivation for locating-dominating codes. We consider these codes in binary hypercubes and generalize the concept for the situation in which we want to locate more than one malfunctioning processor.

Keywords: Locating-dominating codes, Hamming space, Identifying codes

\section{Introduction and definitions}

Let $F=\{0,1\}$ denote the binary field and $F^{n}$ the $n$-dimensional Cartesian product of it. A code is a subset of $F^{n}$. The elements of $F^{n}$ (resp. a code) are called words (resp. codewords) and the Hamming distance $d(x, y)$ between two words $x, y \in F^{n}$ is the number of coordinate positions in which they differ. The Hamming weight $w(x)$ of a word $x \in F^{n}$ is the number of 1 's in $x$. The minimum distance of a code is the smallest distance between any distinct pair of codewords (if the code consists of less than two codewords, then we define the minimum distance to be $n+1$ ). We denote $S_{i}(x)=\left\{y \in F^{n} \mid d(x, y)=i\right\}$ and the Hamming ball $B_{r}(x)=\cup_{i=0}^{r} S_{i}(x)$. If $X \subseteq F^{n}$, we denote by $B_{r}(X)$ the union of the sets $B_{r}(x)$ for $x \in X$. The cardinality of $X$ is denoted by $|X|$ and $V(n, r)=\left|B_{r}(x)\right|$. We say that $x$ r-covers or covers $y$ if $d(x, y) \leq r$.

Consider the binary hypercube (or an arbitrary graph). Assume that it is used to model a multiprocessor architecture, and that each node corresponds to a processor and each edge corresponds to a dedicated link between two processors. We choose some of the processors as codewords, and ask each of them to check its $r$-neighbourhood. Each codeword sends us the symbol 2 if it itself is malfunctioning, 1 if it itself is fine, but at least one processor in its $r$ neighbourhood is malfunctioning, and 0 otherwise. When we have received the reports from all the codewords we wish to locate the malfunctioning processors, under the assumption that

\footnotetext{
†The research of this author was supported by the Academy of Finland under Grants \#44002 and \#200213.

¥The research of this author was supported by the Academy of Finland under Grants \#46186 and \#207303. 
there are at most $l$ of them. The integers $r$ and $l$ are given at the outset. This is the classical locating-dominating set problem $[8]$ if $l=1$ (and then the requirement for the code $C$, i.e., the set of codewords, is that the sets $B_{r}(x) \cap C$ are different and nonempty for all $x \in F^{n} \backslash C$ ). This leads to the following definition.

Definition 1. Let $r$ and $l$ be non-negative integers. $A$ code $C \subseteq F^{n}$ is an $(r, \leq l)$-locatingdominating code of type $A-$ an $(r, \leq l)$-LDA for short - if for all $X \subseteq F^{n}$ and $Y \subseteq F^{n}$ of size at most l,

$$
\begin{gathered}
B_{r}(X) \cap C=B_{r}(Y) \cap C \\
X \cap C=Y \cap C
\end{gathered}
$$

implies that $X=Y$.

If $X=\emptyset$, then $B_{r}(X) \cap C=\emptyset$ : so, in particular, the previous definition implies that $B_{r}(Y) \cap C \neq$ $\emptyset$ whenever $Y \neq \emptyset$.

In the second variant we only consider subsets $X$ and $Y$ of $F^{n} \backslash C$. Then $X \cap C$ and $Y \cap C$ are empty, (2) becomes void, and (1) takes the simple form $B_{r}(X) \cap C=B_{r}(Y) \cap C$. This corresponds to a two-step testing procedure: we first fix all the codewords that have sent 2, and then immediately perform a second round, during which we only receive 1's and 0's.

Definition 2. Let $r$ and $l$ be non-negative integers. $A$ code $C \subseteq F^{n}$ is an $(r, \leq l)$-locatingdominating code of type $B-$ an $(r, \leq l)-L D B$ for short - if for all sets $X \subseteq F^{n} \backslash C$ and $Y \subseteq F^{n} \backslash C$ of size at most $l$,

implies that $X=Y$.

$$
B_{r}(X) \cap C=B_{r}(Y) \cap C
$$

When $l=1$, both definitions reduce to the definition of an $r$-locating-dominating code. Such codes have been considered, for instance, in Haynes, Hedetniemi and Slater [8] and [19, 16, 20, [21, 4, 3].

The smallest cardinality of an $(r, \leq l)$-LDA (resp. an $(r, \leq l)$-LDB) of length $n$ is denoted by $L A_{r}^{(\leq l)}(n)\left(\right.$ resp. $\left.L B_{r}^{(\leq l)}(n)\right)$.

Both these concepts are closely related to that of an identifying code: by definition, a code $C \subseteq F^{n}$ is $(r, \leq l)$-identifying, if for all $X \subseteq F^{n}$ and $Y \subseteq F^{n}$ of size at most $l$, the condition $B_{r}(X) \cap C=B_{r}(Y) \cap C$ implies that $X=Y$. This corresponds to the situation where each codeword only reports back whether it has detected problems in its $r$-neighbourhood or not (and each reply is therefore 0 or 1 ). The minimum cardinality of an $(r, \leq l)$-identifying code of length $n$ is denoted by $M_{r}^{(\leq l)}(n)$. Identifying codes were introduced by Karpovsky, Chakrabarty and Levitin [12] in 1998. Identifying codes in binary hypercubes have been further studied, for instance, in [1, 2, 10, 11, 9, 14]. For results about identifying codes in other graphs, see, e.g., [6. 7.

Clearly, an $(r, \leq l)$-identifying code is an $(r, \leq l)$-LDA, and an $(r, \leq l)$-LDA is an $(r, \leq l)$-LDB.

Example 1. (i) Let $C$ be the even-weight code of length $n \geq 5$, i.e., it consists of all $2^{n-1}$ words of length $n$ that have even weight. An immediate consequence of Theorem 1 is that $C$ a $(1, \leq\lfloor(n+1) / 2\rfloor)$-LDA. Clearly, $C$ is not $(1, \leq 2)$-identifying (take $X=\{100 \ldots 0\}, Y=$ $\{000 \ldots 0,100 \ldots 0\})$. 
(ii) The code $C=F^{n-1} \oplus\{0\}, n \geq 2$, is a $\left(1, \leq 2^{n-1}\right)$-LDB (and hence a $(1, \leq l)$-LDB for all $l$ ). Clearly, $C$ is not $(1, \leq 2)$-LDA (take $X=\{(x, 0)\}$ and $Y=\{(x, 0),(x, 1)\}$ for any $x \in F^{n-1}$ ).

(iii) An $(r, \leq l)$-LDA (resp. an $(r, \leq l)$-LDB) is always an $(r, \leq k)$-LDA (resp. an $(r, \leq k)$-LDB) for all $k \leq l$.

In what follows we often utilize the following lemma, which is easy to check.

Lemma 1. For $a, b \in F^{n}$ we have

$$
\left|B_{1}(a) \cap B_{1}(b)\right|= \begin{cases}n+1 & \text { if } a=b \\ 2 & \text { if } d(a, b)=1 \text { or } 2 \\ 0 & \text { otherwise. }\end{cases}
$$

In Sections 2 and 3 we examine LDAs and LDBs, respectively, when $l \geq 2$. Section 4 deals with the case $l=1$ and the last section considers linear codes. In this paper, we study mainly the case $r=1$.

\section{Bounds and constructions on LDAs}

We have the following characterization of the LDAs; cf. [13].

Theorem 1. Assume that $l \geq 3$. A code $C \subseteq F^{n}$ is a $(1, \leq l)-L D A$ if and only if every element of $F^{n} \backslash C$ is covered by at least $2 l-1$ codewords of $C$.

Proof. Assume first that every element of $F^{n} \backslash C$ is covered by at least $2 l-1$ codewords of $C$. Assume that $X$ and $Y$ are two subsets of $F^{n}$, each of size at most $l$, and that $X \cap C=Y \cap C$ and

$$
B_{1}(X) \cap C=B_{1}(Y) \cap C .
$$

Assume that $x \in X \backslash C$ does not belong to $Y$. Without loss of generality $x=000 \ldots 0$. As a non-codeword, $x$ is covered by at least $2 l-1$ codewords, say $c_{1}, \ldots, c_{2 l-1}$; and without loss of generality, they have been indexed in such a way that $c_{1}, \ldots, c_{i} \in X$, whereas $c_{i+1}, \ldots$, $c_{2 l-1} \notin X(0 \leq i \leq l-1)$. The fact that $X \cap C=Y \cap C$ immediately implies that $c_{1}, \ldots, c_{i} \in Y$ and $c_{i+1}, \ldots, c_{2 l-1} \notin Y$.

By (3), the codewords $c_{i+1}, \ldots, c_{2 l-1}$ are therefore covered by the at most $l-i$ words of weight two of $Y$, each covering at most two of the codewords $c_{i+1}, \ldots, c_{2 l-1}$. Because $2(l-i-1)<$ $2 l-1-i$, there are exactly $l-i$ words of weight two in $Y$ (together with the remaining $i$ words of weight one).

We first notice that the set $X$ does not contain any word of weight four. Indeed, because $X \cap C=Y \cap C$, and $Y$ does not contain any word of weight four, there is no codeword of weight four in $X$. Any non-codeword of weight four in $X$ would be covered by at least $(2 l-1)-4$ $(>0)$ codewords of weight five, which cannot be, because no word in $Y$ could cover a codeword of weight five.

Assume that $y \in Y \backslash C$ has weight two. We show that $y \in X$. By assumption, $y$ is covered by at least $2 l-1$ codewords, of which at least $2 l-3$ have weight three; and they all must be covered by the words in $X$. Because there are no words of weight four in $X$, all these at least $2 l-3$ words of weight three must be covered by the at most $l-1-i$ words of weight two and three in $X$. 
Because $2 l-3>l-1-i$ for all $l \geq 3$, and no word of weight two or three other than $y$ itself covers more than one of these at least $2 l-3$ words, we conclude that $y$ must belong to $X$.

We have shown that $Y \backslash C \subseteq X$. We already knew that $Y \cap C=X \cap C \subseteq X$, and hence $Y \subseteq X$. But $Y$ has size $l$, and therefore $Y=X$, contradicting the fact that $x \in X \backslash Y$.

We have therefore shown that $C$ is a $(1, \leq l)$-LDA.

Assume conversely that $C$ is a $(1, \leq l)$-LDA, and let $x \in F^{n} \backslash C$ be arbitrary. Without loss of generality, $x=000 \ldots 0$. Assume that the codewords that cover $x$ are $c_{1}, c_{2}, \ldots, c_{j}$. Let $i=\lceil j / 2\rceil$. We can choose $i$ words $x_{1}, x_{2}, \ldots, x_{i} \in F^{n} \backslash\{x\}$ that together cover all the words $c_{1}$, $c_{2}, \ldots, c_{j}$. Then $X=\left\{x_{1}, x_{2}, \ldots, x_{i}, x\right\}$ and $Y=\left\{x_{1}, x_{2}, \ldots x_{i}\right\}$ satisfy (1) and (2), and both have cardinality at most $i+1 \leq l$, unless $j \geq 2 l-1$. Hence every non-codeword is covered by at least $2 l-1$ codewords of $C$.

Let us denote by $K(n, r, \mu, \nu)$ the smallest cardinality of a code $C \subseteq F^{n}$ such that every noncodeword is $r$-covered by at least $\mu$ codewords and every codeword is $r$-covered by at least $\nu$ codewords.

From [17, proof of Theorem 1] we get the following lower bound.

Theorem 2. For $l \geq 3$,

$$
L A_{1}^{(\leq l)}(n)=K(n, 1,2 l-1,1) \geq \frac{(2 l-1) 2^{n}}{n+2 l-1} .
$$

Proof. By the previous theorem it suffices to prove the inequality. Assume that $C \subseteq F^{n}$ attains the bound $K(n, 1,2 l-1,1)$. We count in two ways the number of pairs $(c, x)$, where $c \in C$, $x \in F^{n}$ and $d(c, x) \leq 1$. Given $c$, there are $n+1$ choices for $x$, so the number of such pairs equals $(n+1)|C|$. Given $x$, there are at least $2 l-1$ choices for $c$ if $x$ is not a codeword, and at least one, if $x$ is a codeword. Hence $(n+1)|C| \geq(2 l-1)\left(2^{n}-|C|\right)+|C|$, which gives the claim.

From [13] we know that

$$
\frac{M_{1}^{(\leq l)}(n)}{(2 l-1) 2^{n} /(n+1)} \rightarrow 1
$$

for a given $l \geq 3$ when $n \rightarrow \infty$. Because $L A_{1}^{(\leq l)}(n) \leq M_{1}^{(\leq l)}(n)$, the previous theorem gives the following corollary.

Corollary 1. For a fixed $l \geq 3$,

$$
L A_{1}^{(\leq l)}(n) \sim \frac{(2 l-1) 2^{n}}{n}
$$

when $n \rightarrow \infty$.

We also get the following infinite family of exact values.

Theorem 3. Assume that $l \geq 3$. Then

$$
L A_{1}^{(\leq l)}\left((2 l-1)\left(2^{s}-1\right)\right)=2^{(2 l-1)\left(2^{s}-1\right)-s}
$$

for all $s=1,2, \ldots$. 
Proof. From [15] we know that for $n=(2 l-1)\left(2^{s}-1\right)$ the inequality of Theorem 2 is attained by a linear code whose parity check matrix has as its columns $2 l-1$ copies of every non-zero element in $F^{s}$; cf. the beginning of Section 5 .

The direct sum of the codes $C_{1} \subseteq F^{n_{1}}$ and $C_{2} \subseteq F^{n_{2}}$ is

$$
C_{1} \oplus C_{2}=\left\{\left(c_{1}, c_{2}\right) \mid c_{1} \in C_{1}, c_{2} \in C_{2}\right\} \subseteq F^{n_{1}+n_{2}} .
$$

Theorem 4. Let $l \geq 3$. If $C$ is a $(1, \leq l)-L D A$ then the direct sum $C \oplus F$ is also $a(1, \leq l)-L D A$. Thus $L A_{1}^{(\leq l)}(n) \leq 2 L A_{1}^{(\leq l)}(n-1)$.

Proof. The claim follows from Theorem 1 since the direct sum with $F$ preserves the property that each non-codeword is covered by at least $2 l-1$ codewords.

\section{Bounds and constructions on LDBs}

Let us first give a lower bound on the cardinality of a $(1, \leq l)$-LDB for $l \geq 2$. Because trivially $L B_{1}^{(\leq l)}(1)=1$ and $L B_{1}^{(\leq l)}(2)=2$, we may assume in what follows that $n \geq 3$.

Let $C \subseteq F^{n}$. In the sequel, when there is no ambiguity on which code is considered, it is convenient to denote $\left(X \subseteq F^{n}\right)$

$$
I(X)=\bigcup_{x \in X}\left(C \cap B_{1}(x)\right) .
$$

This is called the $I$-set of $X$ and we denote $I\left(\left\{v_{1}, \ldots, v_{s}\right\}\right)=I\left(v_{1}, \ldots, v_{s}\right)$.

We denote

$$
\begin{gathered}
N=F^{n} \backslash C, \\
C_{i}=\{c \in C|| I(c) \mid=i\}, \\
N_{i}=\{x \in N|| I(x) \mid=i\}
\end{gathered}
$$

and $C_{i, j}=\cup_{k=i}^{j} C_{k}$ and further $N_{i, j}=\cup_{k=i}^{j} N_{k}$.

Theorem 5. Let $2 \leq l \leq n-1$. Then

$$
L B_{1}^{(\leq l)}(n) \geq\left\lceil\frac{l}{n+l-1} 2^{n}\right\rceil .
$$

Proof. Assume that $C \subseteq F^{n}$ is a $(1, \leq l)$-LDB where $2 \leq l \leq n-1$.

Let us count in two ways the number of pairs $(x, c)$ where $x \in N_{1, l-1}, c \in C_{n}$ and $d(c, x)=1$. Every $x \in N_{1, l-1}$ has at least one $c \in C_{n}$ in $I(x)$, since otherwise there would exist $y_{i} \in$ $N \cap S_{2}(x) \cap B_{1}\left(c_{i}\right)$ for all $c_{i} \in I(x)$ and thus

$$
I\left(y_{1}, \ldots, y_{|I(x)|}\right)=I\left(y_{1}, \ldots, y_{|I(x)|}, x\right)
$$

which is a contradiction, since $|I(x)| \leq l-1$. On the other hand, each $c \in C_{n}$ can have at most one $x \in N_{1, l-1}$ at distance one from it. Therefore,

$$
\left|N_{1, l-1}\right| \leq\left|C_{n}\right| \text {. }
$$


Next we compute in two ways the number of pairs $(x, c)$ where $c \in C_{1}, x \in N_{l+1, n}$ and $d(x, c)=1$. All the non-codewords at distance one from $c \in C_{1}$ belong to $N_{l+1, n}$. Indeed, assume that there were a word $x$ at distance one from $c$ such that $I(x)=\left\{c_{1}, \ldots, c_{i}, c\right\}(0 \leq i \leq l-1)$. If $i=0$, let $y$ be any word other than $x$ which is at distance 1 from $c$. Then $I(y)=I(y, x)$. Assume that $i \geq 1$. Denoting by $y_{i}$ the unique word other than $x$ such that $d\left(c, y_{i}\right)=d\left(y_{i}, c_{i}\right)=1$, we get the contradiction (note that $y_{i} \in N$ )

$$
I\left(y_{1}, \ldots, y_{i}\right)=I\left(y_{1}, \ldots, y_{i}, x\right) .
$$

On the other hand, a non-codeword $x \in N_{l+1, n}$ can have at most $n$ elements of $C_{1}$ at distance one from it. Consequently,

$$
\left|C_{1}\right| \leq\left|N_{l+1, n}\right| .
$$

Finally, we count the number of pairs $(x, c)$ such that $x \in F^{n}, c \in C$ and $d(x, c) \leq 1$. Now using (4) and the previous inequality, we obtain

$$
\begin{aligned}
|C|(n+1) & =\sum_{i=1}^{n} i\left|N_{i}\right|+\sum_{i=1}^{n+1} i\left|C_{i}\right| \\
& \geq l|N|-(l-1)\left|N_{1, l-1}\right|+\left|N_{l+1, n}\right|+2|C|-\left|C_{1}\right|+(n-2)\left|C_{n}\right| \\
& \geq l|N|+2|C| .
\end{aligned}
$$

This yields the claim.

Theorem 6. If $n \geq 3$ and $l \geq n-1$, then

$$
L B_{1}^{(\leq l)}(n)=2^{n-1} .
$$

Proof. By Example 1 (iii) and the previous theorem, $L B_{1}^{(\leq l)}(n) \geq L B_{1}^{(\leq n-1)}(n) \geq 2^{n-1}$. The claim now follows from Example 1 (ii).

Theorem 7. If $C$ is $a(1, \leq l)-L D B$, then $C \oplus F$ is also $a(1, \leq l)-L D B$. In particular,

$$
L B_{1}^{(\leq l)}(n+1) \leq 2 L B_{1}^{(\leq l)}(n) .
$$

Proof. Suppose $C$ is a $(1, \leq l)$-LDB of length $n$. Divide the words of $F^{n+1}$ into two layers $O_{0}$ and $O_{1}$ depending on the last bit of the word, i.e., $O_{i}=F^{n} \oplus\{i\}$ for $i \in F$. Only a codeword can have in its I-set a codeword from the other layer than where it itself lies. Thus the I-set of a set of non-codewords on the layer $O_{i}$ has codewords only from the same layer. So, if $|X| \leq l$, then $\left|X \cap O_{i}\right| \leq l$ for both $i \in F$, and because $C$ is a $(1, \leq l)$-LDB, $I\left(X \cap O_{i}\right) \subseteq O_{i}$ uniquely identifies $X \cap O_{i}$ for both $i \in F$.

The covering radius of a code $C \subseteq F^{n}$ is defined to be

$$
\max _{x \in F^{n}} \min _{c \in C} d(x, c) .
$$


We denote by $K(n, R)$ the smallest cardinality of a code $C \subseteq F^{n}$ such that it has covering radius at most $R$. For an account of this subject, consult [5].

We denote by $A(n, d, w)$ the smallest cardinality of a code of length $n$ and minimum distance $d$ whose codewords all have weight $w$. For values of this function, see [15].

Denote $S_{i}=S_{i}(00 \ldots 0)$.

Theorem 8. For $l \leq n / 2-1$ we have

$$
L B_{1}^{(\leq l)}(n) \leq K(n, l+1)(V(n, l)-A(n, 6, l)) .
$$

Proof. Let $D$ be a code attaining the value $K(n, l+1), A$ a code attaining the value $A(n, 6, l)$, and $B=B_{l}(00 \ldots 0)$. We shall show that the code

$$
C=D+(B \backslash A)=\{c+b \mid c \in D, b \in B \backslash A\}
$$

is a $(1, \leq l)$-LDB. In the code $C$ there are at most $K(n, l+1)(V(n, l)-A(n, 6, l))$ codewords.

Suppose there are sets $X \subseteq N$ and $Y \subseteq N$ such that $X \neq Y,|X|,|Y| \leq l$ and $I(X)=I(Y)$. There is a word $x \in X$ such that $x \notin Y$. Since $x \notin C$ there exists a codeword $c \in D$ such that $d(x, c)=l$ or $l+1$. Without loss of generality we can assume that $c=00 \ldots 0$.

If $d(x, c)=l$, then $x$ is covered by $l$ codewords of weight $l-1$. None of them can belong to $I(Y)$ since $B_{l-1}(00 \ldots 0) \cap N=\emptyset$ and non-codewords in $S_{l} \backslash\{x\}$ are at least at distance six from $x$.

Suppose now that $d(x, c)=l+1$. Then $l \leq\left|I(x) \cap S_{l}\right| \leq l+1$. If $y \in Y$ covers a codeword of weight $l$ in $I(x)$, then the weight of $y$ must be $l+1$; and since $y \neq x, y$ can cover only one codeword of weight $l$ in $I(x)$. Because $|Y| \leq l$, this implies that $\left|I(x) \cap S_{l}\right|=l,|Y|=l, Y \subseteq S_{l+1}$, and each word in $Y$ covers one codeword from $I(x) \cap S_{l}$.

If $y \in Y$, then $\left|I(y) \cap S_{l}\right|=l+1$, because the minimum distance of non-codewords of weight $l$ is six. As we have already seen (for $x$ whose role $y$ now assumes), this is only possible if $y \in X$. Consequently, $X$ must contain all the $l$ elements of $Y$ together with $x \notin Y$, which is impossible, since $|X| \leq l$.

The previous theorem is interesting in the light of the well-known conjecture [5, p. 352] that for every fixed $R$

$$
\lim _{n \rightarrow \infty} \frac{K(n, R) V(n, R)}{2^{n}}=1 .
$$

Namely, for every fixed $l \geq 2$ Theorems 5 and 8 give the bounds

$$
\frac{l}{n} 2^{n}(1+f(n)) \leq L B_{1}^{(\leq l)}(n) \leq \frac{l+1}{n} 2^{n}(1+g(n)),
$$

where both $f(n)$ and $g(n)$ tend to zero when $n \rightarrow \infty$.

Let us next improve on the lower estimate of Theorem 5 for $\sqrt{n+1} \leq l \leq n-1$.

Lemma 2. Let $C \subseteq F^{n}$ be $a(1, \leq l)-L D B$ with $2 \leq l \leq n-1$. If $j$ is a non-negative integer satisfying $l-j \geq 1$ and $l+j \leq n$, then

$$
(l-j)\left|N_{1, l+j}\right| \leq(n-l+j+1)\left|C_{l-j, n-1}\right|+(l-j)\left|C_{n}\right| .
$$


Proof. Let us count the number of pairs $(c, x)$, where $c \in C_{l-j, n}, x \in N_{1, l+j}$ and $d(x, c)=1$, in two ways. As mentioned in the proof of Theorem 5 if $x \in N_{1, l-1}$, then there exists at least one such pair $(c, x)$.

Let us then concentrate on $x \in N_{l, l+j}$. If there is $c \in I(x)$ such that $c \in C_{n}$, then there exists again one sought pair. Assume then that for all $c \in I(x)$ we have $|I(c)| \leq n-1$. Denote by $M_{l, l+j}$ the subset of $N_{l, l+j}$ which consists of all such elements $x$.

Let $x \in M_{l, l+j}$ and without loss of generality $x=00 \ldots 0$. Denote by $P$ a maximal subset of $\left\{y \in N \cap S_{2}|| I(y) \cap I(x) \mid=2\right\}$ such that its minimum distance is at least four. Since $C$ is a $(1, \leq l)$-LDB, we must have

$$
l \leq|I(x)|-|P| .
$$

Indeed, suppose $|I(x)|-|P| \leq l-1$. Denote by $y_{i}$ a word in $N \cap S_{2}$ such that $d\left(y_{i}, c_{i}\right)=1$ for $c_{i} \in R=I(x) \backslash I(P)$. Notice that such words $y_{i}$ exist for every $c_{i}$ because $x \in M_{l, l+j}$. Now this leads to

$$
I\left(P \cup\left\{y_{1}, \ldots, y_{|R|}\right\}\right)=I\left(P \cup\left\{y_{1}, \ldots, y_{|R|}, x\right\}\right)
$$

which is impossible because

$$
\left|P \cup\left\{y_{1}, \ldots, y_{|R|}, x\right\}\right|=|I(x)|-|P|+1 \leq l .
$$

Clearly, by (5), $0 \leq|P| \leq|I(x)|-l \leq j$. Hence, by virtue of (5), $|R|=|I(x)|-2|P| \geq l-|P| \geq$ $l-j$. Since $P$ is maximal, $\left\{y \in S_{2}|| I(y) \cap R \mid=2\right\} \subseteq C$, and consequently, we get at least $l-j$ pairs from every $x \in M_{l, l+j}$.

All in all the number of sought pairs is at least

$$
\begin{aligned}
& \left|N_{1, l-1}\right|+\left|N_{l, l+j} \backslash M_{l, l+j}\right|+(l-j)\left|M_{l, l+j}\right| \\
= & \left|N_{1, l-1}\right|+\left|N_{l, l+j}\right|+(l-j-1)\left|M_{l, l+j}\right| .
\end{aligned}
$$

On the other hand, at distance one from $c \in C_{l-j, n-1}$ (resp. $c \in C_{n}$ ) there are at most $n-l+j+1$ words (resp. one word) of $N$ and hence possibly of $N_{1, l+j}$. Thus the number of sought pairs is at most

$$
(n-l+j+1)\left|C_{l-j, n-1}\right|+\left|C_{n}\right| .
$$

Furthermore, one immediately verifies the modification of (4) that $\left|N_{1, l-1}\right|+\left|N_{l, l+j} \backslash M_{l, l+j}\right| \leq$ $\left|C_{n}\right|$. Multiplying this by $l-j-1$ and adding it to the inequality which we get from the estimates of the number of pairs proves the claim.

Theorem 9. Let $\sqrt{n+1} \leq l \leq n-1$. Denote

$$
k=\min \left\{\left\lfloor\frac{l^{2}-n-1}{1+l+n}\right\rfloor, n-l-1\right\} .
$$

Then

$$
L B_{1}^{(\leq l)}(n) \geq\left\lceil\frac{l+k+1}{n+l+k+1} 2^{n}\right\rceil .
$$


Proof. Let $C \subseteq F^{n}$ be a $(1, \leq l)$-LDB with $\sqrt{n+1} \leq l \leq n-1$. Obviously,

$$
\begin{aligned}
|C|(n+1) \geq & (l+k+1)|N|-(k+1)\left|N_{l, l+k}\right|-(l+k)\left|N_{1, l-1}\right| \\
& +|C|+(l-k-1)\left|C_{l-k, n-1}\right|+(n-1)\left|C_{n}\right| .
\end{aligned}
$$

Since $l \leq n-1$ we get by (4) that

$$
\begin{aligned}
|C|(n+1) \geq & (l+k+1)|N|-(k+1)\left|N_{l, l+k}\right|-(k+1)\left|N_{1, l-1}\right| \\
& +|C|+(l-k-1)\left|C_{l-k, n-1}\right|+(n-l)\left|C_{n}\right| .
\end{aligned}
$$

The choice of $k$ satisfies

$$
l-k-1 \geq \frac{(k+1)(n-l+k+1)}{l-k}
$$

and furthermore

$$
n-l \geq k+1
$$

Consequently, it is easy to check that we can use Lemma 2 for $j=k$ and hence

$$
|C|(n+1) \geq(l+k+1)|N|+|C| .
$$

The claim follows immediately from this.

If $l \geq \frac{1}{2}\left(\sqrt{2 n^{2}+2 n+1}-1\right), n \geq 3$, then $k=n-l-1$ and the previous theorem combined with Example 1(ii) and Theorem 6 gives the following result.

Theorem 10. Let $n \geq 3$. If $l \geq \frac{1}{2}\left(\sqrt{2 n^{2}+2 n+1}-1\right)$, then

$$
L B_{1}^{(\leq l)}(n)=2^{n-1} .
$$

Next we construct some $(1, \leq 2)$-LDBs.

Theorem 11. Let $n \geq 5$ and $C$ be a code attaining the bound $K(n, 3)$. Then the code

$$
D=C \cup\left\{c+x \mid c \in C, x \in F^{n}, w(x)=2\right\}
$$

is a $(1, \leq 2)-L D B$ and has cardinality at most $K(n, 3)\left(1+\left(\begin{array}{l}n \\ 2\end{array}\right)\right)$.

Proof. Suppose there are $X, Y \subseteq F^{n} \backslash D$ such that $|X|,|Y| \leq 2, X \neq Y$ and $I(X)=I(Y)$. By the definition of $D$, every $y \notin D$ has distance 1 or 3 to $C$.

We first claim that any word $y \in Y$ for which there is a codeword $c \in C$ such that $d(c, y)=1$ also belongs to $X$. Indeed, such a word $y$ is covered by $n(\geq 5)$ codewords of $D$ and thus by Lemma 1 no set of size at most two where $y$ does not belong to can cover all those words. Thus in order to have $I(X)=I(Y)$ we must also have $y \in X$.

Since $X \neq Y$, we can assume that $Y$ contains a word $y \notin X$, and we can assume that $d(y, c)=3$, where $c \in C$. Without loss of generality, $c=0000 \ldots$ and $y=1110 \ldots$ Then $y$ is covered by the codewords $1100 \ldots, 1010 \ldots$ and $0110 \ldots$ of $D$. If an element $x$ of $X \subseteq F^{n} \backslash D$ covers at least one of these three words, then $x$ has weight one or three. If $w(x)=3$, then $x(\neq y)$ covers at most 
one of them. Consequently, there is a word $x \in X$ of weight one which covers two of them. But being of weight one, $x$ must also belong to $Y$. Without loss of generality, $x=1000 \ldots$.

Still we have one codeword $c=0110 \ldots$ which is not in $I(x)$. To cover this word $c$ we need one more word in the set $X$. This word cannot have weight one since then it should belong to $Y$ as well, which is impossible. So, without loss of generality, the other word in $X$ is $0111 \ldots$ But then $0101 \ldots$ and $0011 \ldots$ belong to $I(X)$ but not to $I(Y)$, which gives the required contradiction.

Example 2. The repetition code $\{0000000,1111111\}$ has the properties of Theorem 11. Thus we can construct a $(1, \leq 2)$-LDB of length 7 and cardinality 44 . From this we get by Theorem 7 a $(1, \leq 2)$-LDB of length 8 and cardinality 88 . We know by 18 that $M_{1}^{(\leq 2)}(7)=48$, and the best known $(1, \leq 2)$-identifying code of length 8 is of cardinality 96 .

Let $C$ be the perfect binary Golay code of length 23, dimension 12 and minimum distance 7 (and covering radius 3 ). By Theorem 11 we can construct a $(1, \leq 2)$-LDB of length 23 with cardinality $2^{12}\left(1+\left(\begin{array}{c}23 \\ 2\end{array}\right)\right)=1040384$. It is known [18] that $M_{1}^{(\leq 2)}(23)=2^{20}=1048576$.

\section{Theorem 12.}

$$
L B_{1}^{(\leq 2)}(6) \leq 29 .
$$

Proof. The code $C=\{000000,110000,101000,100100,010100,010001,001010,001001,000110$, 000011, 110010, 100101, 100011, 011100, 011010, 001101, 111010, 111001, 110011, 101110, 101101, $100111,011110,011101,010111,111101,111011,110111,011111\}$ is a $(1, \leq 2)$-LDB of cardinality 29 and length 6.

\section{On the case $l=1$}

In this section we examine the case $l=1$. We denote

$$
L(n)=L A_{1}^{(\leq 1)}(n)=L B_{1}^{(\leq 1)}(n) .
$$

From [12] we know that $M_{1}^{(\leq 1)}(n) \leq n K(n, 2)$. This implies trivially that $L(n) \leq n K(n, 2)$. Denote by $e_{1}$ the word of weight one whose first coordinate equals one.

Theorem 13. For all $n \geq 4$,

$$
L(n+1) \leq(2 n-1) K(n, 2)
$$

Proof. Let $C \subseteq F^{n}(n \geq 4)$ be a code with covering radius two and $K(n, 2)$ codewords. Denote $C_{1}=\left\{w \in F^{n} \mid w \in S_{1}(c)\right.$ for some $\left.c \in C\right\}$ and $C_{2}=\left\{w \in F^{n} \mid w \in S_{1}(c) \backslash\{c+\right.$ $\left.e_{1}\right\}$ for some $\left.c \in C\right\}$. We claim that $D=C_{1} \oplus\{0\} \cup C_{2} \oplus\{1\}$ is 1-locating-dominating.

We again denote $O_{i}=F^{n} \oplus\{i\}$ for $i=0,1$. By the construction, $I(x) \neq \emptyset$ for all $x \in F^{n+1}$. Assume that $x, y \in F^{n+1} \backslash D$ and $x \neq y$. It is shown in [12] that $C_{1}$ is $(1, \leq 1)$-identifying. Hence, if $x, y \in O_{0}$, then $I(x) \neq I(y)$.

Suppose $x \in O_{0}$ and $y \in O_{1}$. Because $x \notin D$, the structure of $C_{1}$ implies that $\left|I(x) \cap O_{0}\right| \geq 2$ whereas $\left|I(y) \cap O_{0}\right| \leq 1$. Hence again $I(x) \neq I(y)$. 
Assume next that $x, y \in O_{1} \backslash D$. There exists $c \in C \oplus\{1\}$ such that $d(c, x) \leq 2$. If $x=c$, then $\left|I(x) \cap S_{1}(c)\right| \geq 3$ and $|I(y) \cap I(x)| \leq 2$ by Lemma 1. If $x=c+e_{1}$, then $I(x) \cap O_{0} \neq \emptyset$ and thus $I(x) \neq I(y)$. Assume then that $x \in S_{2}(c)$ and that $x$ covers two elements of $I(c)$. In this case only $y=c$ could cover both elements of $I(x) \cap S_{1}(c)$, and this is impossible as we have seen. Suppose finally that $x \in S_{2}(c)$ and only covers one element of $I(c)$ : in other words, that $c+e_{1} \notin D, d\left(x, c+e_{1}\right)=1$. Then $y$ should cover the unique element in $I(x) \cap S_{1}(c)$ and consequently $y \in S_{2}(c)$, but then $y$ would cover also another codeword of $I(c)$, which gives $I(x) \neq I(y)$.

By [20, Theorem 10] we have the next theorem.

\section{Theorem 14.}

$$
L(n) \geq\left\lceil\frac{2^{n+1}}{n+3}\right\rceil
$$

Let us recall the notion of excess (see, e.g., [5, Chapter 6], 22]). Assume that the covering radius of $C \subseteq F^{n}$ is at most 1 . We denote the excess on $x \in F^{n}$ by $E(x)=|I(x)|-1$ and

$$
E(X)=\sum_{x \in X} E(x)
$$

Theorem 15.

$$
L(n) \geq\left\lceil\frac{n^{2} 2^{n+1}}{n^{3}+2 n^{2}+3 n-2}\right\rceil .
$$

Proof. (Cf. 11.) Suppose $C$ is an optimal 1-locating-dominating code of length $n$. Denote by $K$ the cardinality of $C$.

It is not difficult to check that there can be only four kinds of words in $F^{n}$ :

1. a word which is covered by exactly one codeword,

2. a codeword, say $c_{1}$, which forms a couple with another codeword $c_{2}$ such that $I\left(c_{1}\right)=$ $I\left(c_{2}\right)=\left\{c_{1}, c_{2}\right\}$,

3. a word $x$ which has $E(x) \geq 2$, and

4. a word $y$ which has $E(y)=1$ and for which there exists a word $x$ such that $E(x) \geq 2$ and $I(y) \subset I(x)$.

A word of type 3 is called a father. A word of type 4 is called a son; the word $x$ such that $I(y) \subset I(x)$ is called its father (it is easy to see that $x$ is uniquely defined). A family consists of a father and its sons. The families, couples and points with excess zero partition the whole space $F^{n}$.

Suppose that a father is covered by $i$ codewords. Then there are at most $\left(\begin{array}{l}i \\ 2\end{array}\right)$ sons in the family. The average excess on the points in a family whose father is covered by exactly $i \geq 3$ codewords is therefore at least

$$
f(i):=\frac{\left(\begin{array}{l}
i \\
2
\end{array}\right)+i-1}{\left(\begin{array}{l}
i \\
2
\end{array}\right)+1}
$$


This is decreasing function on $i \geq 4$; and $f(3)=f(6)$. Assume that $n \geq 6$. Then $f(i) \geq f(n)$ for all $3 \leq i \leq n$. Since $C$ is an optimal code there is no codeword $c \in C$ such that $|I(c)|=n+1$.

The excess on $F^{n}$ is $K(n+1)-2^{n}$. We now estimate it in a different way. There are at most $2 K$ words outside the families. We can estimate

$$
K(n+1)-2^{n} \geq\left(2^{n}-2 K\right) f(n),
$$

from which we get the claimed lower bound on $K$ for $n \geq 6$.

When $n=5$ then the minimum of $f(3), f(4)$ and $f(5)$ is $f(3)$. Now we can estimate $6 K-2^{5} \geq$ $\left(2^{5}-2 K\right) f(3)$. From this we get $K \geq 9$. And thus the lower bound holds for $n=5$. Similarly when $n=4$ we notice that the lower bound holds. For $1 \leq n \leq 3$ the lower bound is true by Theorem 14.

Theorem 16. If $C$ is 1-locating-dominating with the property that $d(c, C \backslash\{c\})=1$ for all $c \in C$, then $D=C \oplus F \subseteq F^{n}$ is $(1, \leq 1)$-identifying.

Proof. Assume that $x, y \in F^{n}, x \neq y$. We show that $I(x) \neq I(y)$.

We again denote $O_{i}=F^{n-1} \oplus\{i\}$.

Assume first that $x, y \in O_{0}$ (the case $x, y \in O_{1}$ can be proved in the same way). If $x, y \notin D$, then they can be separated (their $I$-sets are different) by the locating-dominating property of $C$. If $x \in D$, but $y \notin D$, then $I(x) \cap O_{1} \neq \emptyset$ whereas $I(y) \cap O_{1}=\emptyset$. If both $x, y \in D$ and $I(x)=I(y)$, then $I(x) \cap O_{1}=I(y) \cap O_{1}$ and thus $x=y$.

Suppose then that $x \in O_{0}$ and $y \in O_{1}$. If $x \notin D$, then $I(x) \cap O_{1}=\emptyset$ and we are done. Assume then that $x \in D$. By the assumption $d(x,(C \oplus\{0\}) \backslash\{x\})=1$, and hence $\left|I(x) \cap O_{0}\right| \geq 2$ and $\left|I(y) \cap O_{0}\right| \leq 1$.

The bounds on $L B_{1}^{(\leq l)}(n)$ for small values of $l$ and $n$ are summarized in Table 1 . The cases $n=1$ are trivial. The codes $\{0000,1100,0101,1110,1011,0111\}$ and $\left\{x \in F^{5} \mid w(x)=1\right.$ or 4$\}$ are 1-locating-dominating. All the other upper bounds are from Example 1(ii). The lower bounds $L(2) \geq 2$ and $L(3) \geq 4$ are easy to check by hand and Example 1 (iii) then gives the other lower bounds for lengths two and three. The lower bounds on $L(4), L(5)$ and $L B_{1}^{(\leq 2)}(4)$ have been obtained by computer, $L B_{1}^{(\leq 3)}(4) \geq 8$ and $L B_{1}^{(\leq 2)}(5) \geq 11$ from Theorem 5 and $L B_{1}^{(\leq 3)}(5) \geq 15$ from Theorem 9 ,

\begin{tabular}{|c|c|c|c|}
\hline$n$ & $L(n)$ & $L B_{1}^{(\leq 2)}(n)$ & $L B_{1}^{(\leq 3)}(n)$ \\
\hline 1 & 1 & 1 & 1 \\
\hline 2 & 2 & 2 & 2 \\
\hline 3 & 4 & 4 & 4 \\
\hline 4 & 6 & 8 & 8 \\
\hline 5 & 10 & $11-16$ & $15-16$ \\
\hline
\end{tabular}

Tab. 1: Values of $L B_{1}^{(\leq l)}(n)$ for short lengths and $l=1,2,3$. 


\section{Optimal linear locating-dominating codes}

A binary code $C \subseteq F^{n}$ is said to be linear if it is a $k$-dimensional subspace of $F^{n}$. In this section we consider linear $(1, \leq l)$-LDBs and $(1, \leq l)$-LDAs. We denote by $L B_{1}^{(\leq l)}[n]\left(\operatorname{resp} . L A_{1}^{(\leq l)}[n]\right)$ the smallest cardinality of a linear $(1, \leq l)$-LDB (resp. $(1, \leq l)$-LDA) of length $n$.

A linear $k$-dimensional code $C \subseteq F^{n}$ can always be defined by choosing an $(n-k) \times n$ matrix $H$ - called its parity check matrix - such that

$$
x \in C \text { if and only if } H x^{T}=0 .
$$

Let $x \in F^{n}$ be arbitrary, and consider its syndrome $H x^{T}$. If $H x^{T}$ appears in $H$ as the $i$-th column, then clearly the word obtained by changing the $i$-th bit in $x$ belongs to $C$. Consequently, if $x$ is a codeword, the number of codewords within distance one from it equals one plus the number of zero columns in $H$; if $x$ is a non-codeword, the number of codewords within distance one from it equals the number of times the syndrome of $x$ appears as a column in $H$.

The next auxiliary lemma from [17] will be used often.

Lemma 3. If $C \subseteq F^{n}$ is a linear code and $c \in C$ then for all $x \in F^{n}$

$$
I(x+c)=I(x)+c .
$$

Proof. Clearly, $y \in I(x+c)$ if and only if $y \in C$ and $d(x+c, y) \leq 1$. This is equivalent to the fact that $y+c \in C$ (because $C$ is linear) and $d(x, y+c) \leq 1$; that is, $y+c \in I(x)$. This is equivalent to $y \in I(x)+c$.

Let us first consider the case $l=1$ and denote $L[n]=L A_{1}^{(\leq 1)}[n]=L B_{1}^{(\leq 1)}[n]$.

\section{Theorem 17.}

$$
L[n] \geq 2^{r} \geq\left\lceil\frac{3 \cdot 2^{n}}{n+5}\right\rceil,
$$

where $r$ is the smallest integer such that the latter inequality holds.

Proof. Suppose $C$ is a linear $(1, \leq 1)$-LDB of length $n$ and $|C|=K$. There can be $K$ noncodewords which are covered by one codeword each. All the other non-codewords must be covered by at least three codewords. Namely, if $I(x)=\left\{c_{1}, c_{2}\right\}$ for some $x \notin C$, then for $y=x+c_{1}+c_{2}$, $I(y)=I(x)$ by Lemma 3

Thus we have $2 K+3\left(2^{n}-2 K\right) \leq K(n+1)$, from which the claim follows.

Theorem 18. Let $n=3 \cdot 2^{k}-5+s$, for $k \geq 1$ and $0 \leq s<3 \cdot 2^{k}$. Then $L[n]=2^{n-k}$.

Proof. The lower bound follows from Theorem 17 .

Because $n \geq 3 \cdot 2^{k}-5$, we can choose a $k \times n$ matrix $H$ in which every nonzero column appears at least three times except for one nonzero column which appears exactly once. Let $C$ be the code with parity check matrix $H$. We claim that $C$ is a $(1, \leq 1)$-LDB. Each non-codeword which is covered by at least three codewords is clearly identified since in the intersection of three Hamming balls of radius one there is at most one word. Each non-codeword of $N_{1}$ is also identified, because for each $c \in C$, there is exactly one word of $N_{1}$ at distance one from $c$. 
Lemma 4. Suppose $C$ is $(1, \leq 2)-L D B$ of length $n$ and $x \notin C$. If $I(x)=\{c\}$, then $|I(c)|=n$. If $I(x)=\left\{c_{1}, c_{2}\right\}$ for some $c_{1}, c_{2} \in C, c_{1} \neq c_{2}$, then $x+c_{1}+c_{2} \in C$.

Proof. See the proof of 4 for the first claim.

If $I(x)=\left\{c_{1}, c_{2}\right\}, c_{1} \neq c_{2}$, and $x+c_{1}+c_{2} \notin C$ then $I(x) \subseteq I\left(x+c_{1}+c_{2}\right)$ and hence $I\left(x, x+c_{1}+c_{2}\right)=I\left(x+c_{1}+c_{2}\right)$, which is a contradiction.

Theorem 19. For all $n \geq 5$,

$$
L B_{1}^{(\leq 2)}[n] \geq 2^{r} \geq\left\lceil\frac{5 \cdot 2^{n}}{n+5}\right\rceil,
$$

where $r$ is the smallest integer such that the latter inequality holds.

Proof. Let $C$ be a linear $(1, \leq 2)$-LDB of cardinality $K$.

If $I(x)=\{c\}$ for some $x \notin C$ then by Lemma $4,|I(c)|=n$ and thus by Lemma $3,|I(c)|=n$ for all $c \in C$. Hence $K \cdot n+\left(2^{n}-K\right) \leq K(n+1)$, i.e., $K \geq 2^{n-1}$, and the claim follows. So assume that $|I(x)| \geq 2$ for all $x \notin C$.

Assume that $|I(x)|=2$ for some $x \notin C$, and that $I(x)=\left\{c_{1}, c_{2}\right\}$. Then by Lemma 4 . $x+c_{1}+c_{2} \in C$, which is impossible when $C$ is linear and $x \notin C$ and $c_{1}, c_{2} \in C$.

Similarly, as in [17, Theorem 6] we can show that each non-codeword must be covered by at least five codewords. We repeat the proof here. First we show that $|I(x)| \geq 4$ for all $x \notin C$. Assume on the contrary that $x \notin C$ and $|I(x)|=3$. Without loss of generality (by Lemma 3) we can assume that $x \in S_{1}$. There exist two different non-codewords $y$ and $z$ in $S_{1} \backslash\{x\}$ such that $x+y$ and $x+z$ belong to the code, and by linearity also $y+z \in C$. Now $I(x)=\{00 \ldots 0, x+y, x+z\}$ and by Lemma $3, I(y)=I(x+(x+y))=\{x+y, 00 \ldots 0, y+z\}$ and $I(z)=I(x+(x+z))=$ $\{x+z, y+z, 00 \ldots 0\}$. Hence $I(x, y)=\{00 \ldots 0, x+y, x+z, y+z\}=I(x, z)$, which is a contradiction. Hence $|I(x)| \geq 4$ for all $x \notin C$.

Assume now that $x \notin C$ and $I(x)=\left\{x+e_{1}, x+e_{2}, x+e_{3}, x+e_{4}\right\}$ where $e_{i} \in S_{1}$, for $i=1, \ldots, 4$ are different non-codewords. Consider the non-codewords

$$
\begin{aligned}
& y=x+e_{1}+e_{2}, \\
& z=x+e_{3}+e_{4}, \\
& v=y+e_{3}+e_{4} .
\end{aligned}
$$

Using Lemma 3 we get

$$
\begin{aligned}
& I(y)=\left\{y+e_{1}=x+e_{2}, y+e_{2}=x+e_{1}, y+e_{3}, y+e_{4}\right\}, \\
& I(z)=\left\{z+e_{1}, z+e_{2}, z+e_{3}=x+e_{4}, z+e_{4}=x+e_{3}\right\}, \\
& I(v)=\left\{v+e_{1}, v+e_{2}, v+e_{3}=y+e_{4}, v+e_{4}=y+e_{3}\right\} .
\end{aligned}
$$

Now $z+e_{1}=x+e_{3}+e_{4}+e_{1}=y+e_{3}+e_{4}+e_{2}=v+e_{2}$ and similarly $z+e_{2}=v+e_{1}$. Thus we have $I(x, v)=I(y, z)$ which is a contradiction, and hence $|I(x)| \geq 5$ for all $x \notin C$.

Now we have $K+5\left(2^{n}-K\right) \leq K(n+1)$, from which the claim follows.

Theorem 20. Let $n=5\left(2^{k}-1\right)+s$, for $k \geq 1$ and $0 \leq s<5 \cdot 2^{k}$. Then $L B_{1}^{(\leq 2)}[n]=2^{n-k}$. 
Proof. The lower bound follows from Theorem 19. Let $H$ be a $k \times n$ matrix where every nonzero $k$-tuple appears at least five times, and let $C$ be the code which has parity check matrix $H$. Now every non-codeword is covered by five codewords of $C$, and this code is a $(1, \leq 2)$-LDB. Namely, if $X, Y \subseteq F^{n} \backslash C$ have both size at most two and $x \in X \backslash Y$, then by Lemma 1, the at most two words in $Y$ cannot cover all the at least five codewords in $I(x)$.

Theorem 21. For $l \geq 3$ and $n \geq 2 l-1$,

$$
L B_{1}^{(\leq l)}[n] \geq 2^{r} \geq\left\lceil\frac{(2 l-1) 2^{n}}{n+2 l-1}\right\rceil,
$$

where $r$ is the smallest integer such that the latter inequality holds.

Proof. Assume that $C$ is a linear $(1, \leq l)$-LDB of length $n$ and cardinality $K$.

If there is a word $x \notin C$ such that $|I(x)|=1$, then the beginning of the previous proof (since $C$ is trivially also a $(1, \leq 2)$-LDB) shows that $K \geq 2^{n-1}$, and since we have assumed that $n \geq 2 l-1$, we are already done.

Assume now that $|I(x)| \geq 2$ for all $x \notin C$. We shall show that each non-codeword must be covered by at least $2 l-1$ codewords. Assume to the contrary that $x \notin C,|I(x)|=j$ for some $2 \leq j \leq 2 l-2$, and that $I(x)=\left\{c_{1}, \ldots, c_{j}\right\}$. For all $i, 1 \leq i \leq j / 2$, let $x_{i}=x+c_{2 i-1}+c_{2 i}$ (which is the unique word other than $x$ at distance one from both $c_{2 i-1}$ and $c_{2 i}$ ). If $j$ is odd, define $x_{0}=x+c_{1}+c_{j}$. Let $X$ consist of all the words $x_{i}, 1 \leq i \leq j / 2$, together with $x_{0}$, if $j$ is odd. Because $C$ is linear and $x \notin C$, we know that $X \subseteq F^{n} \backslash C$. Now $I(X)=I(X \cup\{x\})$ gives a contradiction, because $|X \cup\{x\}|=\lceil j / 2\rceil+1 \leq l$.

Thus we have $K+(2 l-1)\left(2^{n}-K\right) \leq K(n+1)$, and the claim follows.

Theorem 22. Let $l \geq 3$ and $n=(2 l-1)\left(2^{k}-1\right)+s$, for $k \geq 1$ and $0 \leq s<(2 l-1) 2^{k}$. Then $L B_{1}^{(\leq l)}[n]=2^{n-k}$.

Proof. The lower bound follows from Theorem 21. Let $H$ be a $k \times n$ matrix in which every nonzero $k$-tuple appears at least $2 l-1$ times. The code which has $H$ as a parity check matrix is $(1, \leq l)$-LDB by Theorem 1 , and has the required dimension.

The previous proof in fact gives the following theorem as an immediate corollary.

Theorem 23. Let $l \geq 3$. For all $n \geq 2 l-1$, the smallest dimension of a linear $(1, \leq l)-L D A$ of length $n$ is the same as the smallest dimension of a linear $(1, \leq l)-L D B$ of length $n$.

Theorem 24. For all $n \geq 5$, the smallest dimension of a linear $(1, \leq 2)$-LDA of length $n$ is the same as the smallest dimension of a linear $(1, \leq 2)-L D B$ of length $n$.

Proof. By Theorems 20, 22 and 23 we know that for all $n \geq 5, L B_{1}^{(\leq 2)}[n]=L B_{1}^{(\leq 3)}[n]=$ $L A_{1}^{(\leq 3)}[n]$. Trivially, $L A_{1}^{(\leq 3)}[n] \geq L A_{1}^{(\leq 2)}[n] \geq L B_{1}^{(\leq 2)}[n]$, and the claim follows. 


\section{References}

[1] U. Blass, I. Honkala, and S. Litsyn: Bounds on identifying codes. Discrete Mathematics, 241(1-3):119-128, 2001.

[2] U. Blass, I. Honkala, and S. Litsyn: On binary codes for identification. Journal of Combinatorial Designs, 8:151-156, 2000.

[3] I. Charon, O. Hudry, and A. Lobstein: Identifying and locating-dominating codes: NPcompleteness results for directed graphs. IEEE Transactions on Information Theory, 48(8):2192-2200, 2002.

[4] I. Charon, O. Hudry, and A. Lobstein: Minimizing the size of an identifying or locatingdominating code in a graph is NP-hard. Theoretical Computer Science, 290:2109-2120, 2003.

[5] G. Cohen, I. Honkala, S. Litsyn, and A. Lobstein: Covering Codes. Elsevier, Amsterdam, 1997.

[6] G. Cohen, I. Honkala, A. Lobstein, and G. Zémor: New bounds for codes identifying vertices in graphs. Electronic Journal of Combinatorics, 6(1)(R19), 1999.

[7] G. D. Cohen, I. Honkala, A. Lobstein, and G. Zémor: On identifying codes. In A. Barg and S. Litsyn, editors, Codes and Association Schemes, Proceedings of the DIMACS Workshop on Codes and Association Schemes, DIMACS Series in Discrete Mathematics and Theoretical Computer Science 56, AMS, Providence, pages 97-109, 2001.

[8] T. W. Haynes, S. T. Hedetniemi, and P. J. Slater: Fundamentals of Domination in Graphs. Marcel Dekker, New York, 1998.

[9] I. Honkala, T. Laihonen, and S. Ranto: On codes identifying sets of vertices in Hamming spaces. Designs, Codes and Cryptography, 24:193-204, 2001.

[10] I. Honkala and A. Lobstein: On identifying codes in binary Hamming spaces. Journal of Combinatorial Theory, Series A, 99(2):232-243, 2002.

[11] I. Honkala and A. Lobstein: On the complexity of the identification problem in Hamming spaces. Acta Informatica, 38:839-845, 2002.

[12] M. G. Karpovsky, K. Chakrabarty, and L. B. Levitin: On a new class of codes for identifying vertices in graphs. IEEE Transactions on Information Theory, 44(2):599-611, 1998.

[13] T. Laihonen: Sequences of optimal identifying codes. IEEE Transactions on Information Theory, 48(3):774-776, 2002.

[14] T. Laihonen and S. Ranto: Codes identifying sets of vertices. In S. Boztaş and I. Shparlinski, editors, Applied Algebra, Algebraic Algorithms and Error-Correcting Codes, Proceedings of $A A E C C-14$, Lecture Notes in Computer Science 2227, Springer, Berlin, pages 82-91, 2001.

[15] E. M. Rains and N. J. A. Sloane: Table of constant weight binary codes. Published electronically at http://www.research.att.com/ njas/codes/Andw/. 
[16] D. F. Rall and P. J. Slater: On location-domination numbers for certain classes of graphs. Congressus Numerantium, 45:97-106, 1984.

[17] S. Ranto: Optimal linear identifying codes. IEEE Transactions on Information Theory, 49:1544-1547, 2003.

[18] S. Ranto, I. Honkala, and T. Laihonen: Two families of optimal identifying codes in binary Hamming spaces. IEEE Transactions on Information Theory, 48(5):1200-1203, 2002.

[19] P. J. Slater: Dominating and reference sets in a graph. Journal Mathematical and Physical Sciences, 22:445-455, 1988.

[20] P. J. Slater: Locating dominating sets and locating-dominating sets. In Y. Alavi and A. Schwenk, editors, Graph Theory, Combinatorics and Applications: Proceedings of the Seventh Quadrennial International Conference on the Theory and Applications of Graphs, volume 2, Wiley, pp. 1073-1079, 1995.

[21] P. J. Slater: Fault-tolerant locating-dominating sets. Discrete Mathematics, 249(1-3):179$189,2002$.

[22] G. J. M. van Wee: Improved sphere bounds on the covering radius of codes, IEEE Transactions on Information Theory, 34: 237-245, 1988. 
